\title{
Robust controller design based on reduced order plants
}

\section{A. B. Özgüler \& A. N. Gündeş}

To cite this article: A. B. Özgüler \& A. N. Gündeș (2006) Robust controller design based on reduced order plants, International Journal of Control, 79:12, 1624-1634, DOI: 10.1080/00207170600892931

To link to this article: http://dx.doi.org/10.1080/00207170600892931

册 Published online: 20 Feb 2007.

Submit your article to this journal

Џll Article views: 90

Q View related articles $₫$

Citing articles: 3 View citing articles 


\title{
Robust controller design based on reduced order plants
}

\author{
A. B. ÖZGÜLER † and A. N. GÜNDEŞ* \\ $†$ Electrical and Electronics Engineering, Bilkent University, Ankara, 06800 Turkey \\ $\$$ Electrical and Computer Engineering, University of California, Davis, CA 95616
}

(Received 25 November 2005; in final form 23 February 2006)

\begin{abstract}
Two dual controller design methods are proposed for linear, time-invariant, multi-input multi-output systems, where designs based on a reduced order plant robustly stabilizer higher order plants with additional poles or zeros in the stable region. The additional poles (or zeros) are considered as multiplicative perturbations of the reduced plant. The methods are tailored towards closed-loop stability and performance and they yield estimates for the stability robustness and performance of the final design. They can be considered as formalizations of two classical heuristic model reduction techniques. One method neglects a plant-pole sufficiently far to the left of dominant poles and the other cancels a sufficiently small stable plant-zero with a pole at the origin.
\end{abstract}

\section{Introduction}

Controllers stabilizing a complex plant and achieving a specified performance are usually at least as complex as the plant itself (Zhou et al. 1996). Both the computation and the implementation of such controllers are serious issues to be dealt with in control system design. There are two main approaches for simplification of the design process: (i) the first is to design the high-order controller and then to approximate it with a low-order one within an acceptable loss of performance; (ii) the second is to reduce the order of the plant model with the prospect that a low-order model will lead to a low-order controller. The drawback of the first approach is that the high-order controller computation problem is not avoided. Hence, there are various efforts to reduce the computational burden as in (Varga 2003) and the references therein. An alternative to (i) is to seek to minimize a closed-loop performance index by a fixed order controller (Ly 1982, Bernstein and Hyland 1985); however, there are many issues to be better understood in such methods as discussed in Anderson and Liu (1989). For the second approach, the main drawback is the difficulty in quantifying the loss of closed-loop performance. This is because a satisfactory approximation of the plant model requires some knowledge of the

*Corresponding author. Email: angundes@ucdavis.edu controller in advance, and an acceptable low-order controller cannot be calculated unless the plant model is specified (Enns 1984). Hence, (ii) can only be used in an iterative scheme, where a reduced plant model is obtained, a controller is designed, performance is evaluated, and these steps are repeated until a satisfactory closed-loop system is obtained.

This paper proposes two dual methods of controller design for reduced order linear, time-invariant, multiinput multi-output (MIMO), stable or unstable systems in the general frame-work of approach (ii) above. The first method neglects poles sufficiently far from dominant poles in the stable region, and the second method reduces the plant order by canceling a zero near the origin with a pole at the origin. The proposed methods come with performance bounds on the closed-loop sensitivity and complementary sensitivity matrices and are iterative in nature. The main idea is based on perhaps the oldest heuristic reduction techniques covered in classical control textbooks (Rohrs et al. 1993, Kuo 1995, Ogata 1997), where controllers are first designed for reduced order plants with the "insignificant" poles deleted or for reduced order plants obtained by deleting a zero "close to the origin" together with a pole at the origin. These two seemingly contradictory methods were shown to be dual model reduction methods in Özgüler and Gündeş (2002). Here, they are formalized as systematic control design methods with 
emphasis on closed-loop performance as well as stability. The main results explicitly define regions such that controllers designed for reduced order plants are guaranteed to stabilize higher order plants with poles (or zeros) in these regions while ensuring an acceptable performance. The advantage is that only the lower order model needs to be known explicitly so that stabilizing controllers can be designed. The poles (or zeros) for the higher order model need not be known, since the controller designed for the lower order model guarantees stability based on regions, not specific points.

Model reduction methods, whether they are used for the purpose of simulation or control, are developed in many different disciplines and are surveyed in Al-Saggaf and Franklin (1988), Anderson and Liu (1989) and Antoulas et al. (2001). Computationally attractive methods such as Padé, modal, or continued-fraction approximations or moment matching methods generally have no guaranteed stability/performance. The balanced realization method (Moore 1981), the Hankel norm approximation method (Adamjan et al. 1971, Kung and Lin 1981, Glover 1984), and the $q$-covariance equivalent method (Yousuff et al. 1985) are among rigorous model reduction methods that come with some kind of a performance criterion. The closed-loop performance of such order reduction methods when used for the purpose of control system design was studied recently. For example some performance bounds for the coprime factor controller reduction method of Anderson and Liu (1989) are given in Enns (1984) the frequency weighted balanced reduction method of Enns (1984) is combined with Anderson and Liu (1989) in (Liu et al. (1990) (see also (Varga 2003)). An interesting but heuristic study of closed-loop balanced reduction is that of Wortelboer et al. (1999), where an iterative procedure for plant and controller reduction in a closed-loop configuration is proposed.

Our main results apply to linear, time-invariant, MIMO continuous-time systems; they apply to discrete-time systems with minor modifications. A narrative description of the proposed order reduction methods and comparisons with some alternative approaches are in $\S 2$. Section 3 contains the main results (the dual Theorems 1 and 2) and several illustrative examples. Concluding remarks are given in $\S 4$. Preliminary versions of these results were presented in Özgüler and Gündeş (2003).

The following notation is used: $\mathbf{S}$ denotes stable proper real rational functions of $s$ (real-rational $H_{\infty}$ functions); $\mathcal{M}(\mathbf{S})$ denotes matrices whose entries are in $\mathbf{S} ; U \in \mathcal{M}(\mathbf{S})$ is unimodular iff $U^{-1} \in \mathcal{M}(\mathbf{S}) ; \mathbf{R}_{\mathbf{p}}$ denotes proper and $\mathbf{R}_{\mathbf{S}}$ denotes strictly-proper rational functions; $\mathcal{M}\left(\mathbf{R}_{\mathbf{p}}\right)$ and $\mathcal{M}\left(\mathbf{R}_{\mathbf{s}}\right)$ denote matrices whose entries are in $\mathbf{R}_{\mathbf{p}}$ and $\mathbf{R}_{\mathbf{s}}$, respectively; $\mathbb{R}, \mathbb{C}, \mathbb{C}_{-}$denote real, complex, and left-half plane complex numbers. The $H_{\infty}$-norm of a matrix $M(s) \in \mathcal{M}(\mathbf{S})$ is denoted by $M(s)$ (i.e., the norm $\|\cdot\|$ is defined as $\|M\|=\sup _{s \in \partial \mathcal{U}} \bar{\sigma}(M(s))$, where $\bar{\sigma}$ denotes the maximum singular value and $\partial \mathcal{U}$ denotes the boundary of the extended closed right-half-plane $\mathcal{U}$ ). For simplicity, we drop $(s)$ in transfer matrices such as $G(s)$.

\section{Preliminaries}

In this section, we describe the proposed methods of designing controllers based on reduced order versions of the plant, and provide a brief comparison with standard robustness approaches. We consider a high-order plant $G_{h}$ and a low-order version $G_{l}$ obtained from $G_{h}$ either (a) by deleting some of its poles, or (b) by cancelling some of its zeros by its poles at the origin (in such poles exist). Our goal is to answer the following questions. Let $H_{l}$ be a stabilizing controller for $G_{l}$. Can we put limitations on the performance of $H_{l}$ in the closed-loop system $\left(G_{l}, H_{l}\right)$ such that it is also a stabilizing controller for $G_{h}$ ? If so, can we estimate a bound on the performance of $\left(G_{h}, H_{l}\right)$ ?

Theorem 1 of $\S 3$ deals with case (a) above. It shows that if $H_{l}$ is a stabilizing controller for $C_{l}$ and achieves a sufficiently quenched complementary sensitivity function for the closed-loop $\left(G_{l}, H_{l}\right)$ at high-frequencies, then $H_{l}$ also stabilizes $G_{h}$ and achieves a complementary sensitivity with similar high-frequency characteristics for the closed-loop $\left(G_{h}, H_{l}\right)$. A lower and upper bound on the $H_{\infty}$-norm the sensitivity of $\left(G_{h}, H_{l}\right)$ is also derived in Theorem 1. Theorem 2 deals with case (b) above. It shows that if $H_{l}$ is a stabilizing controller for $G_{l}$ and achieves a sufficiently quenched sensitivity function for the closed-loop $\left(G_{l}, H_{l}\right)$ at low-frequencies, then $H_{l}$ also stabilizes $G_{h}$ and achieves a sensitivity with similar lowfrequency characteristics for the closed-loop $\left(G_{h}, H_{l}\right)$. A lower and upper bound on the $H_{\infty}$-norm of the complementary sensitivity of $\left(G_{h}, H_{l}\right)$ is also derived in Theorem 2. In both cases, the crucial question of whether $G_{l}$ admits a stabilizing controller with a good enough closed-loop performance can be settled by solving a standard $H_{\infty}$-optimization problem as detailed in Remarks 2 and 8 in $\S 3$.

In both (a) and (b), the high-order plant $G_{h}$ can be regarded as a multiplicatively perturbed version of $G_{l}$ since it can be expressed as $G_{h}=(1-\Delta) G_{l}$ for a stable transfer function $\Delta$ satisfying $\Delta=1$. Although the stability aspect of the order reduction problem can be approached via the existing standard robustness results such as those in Doyle and Stein (1981) there are two problems with this perturbation approach. Let $T_{l}=G_{l} H_{l}\left(I+G_{l} H_{l}\right)^{-1}$ be the complementary sensitivity matrix associated with $\left(G_{l}, H_{l}\right)$. By Doyle and Stein (1981) (also Anderson and Liu (1989)), 
$H_{l}$ stabilizes $G_{h}$ if $\left\|\Delta T_{l}\right\|<1$. First, since $\|\Delta\|=1$ is independent of insignificant poles/zeros, writing $\left\|\Delta T_{l}\right\| \leq\|\Delta\|\left\|T_{l}\right\|=\left\|T_{l}\right\|<1$ simply says that closedloop stability is guaranteed regardless of the candidate insignificant poles/zeros if $\left\|T_{l}\right\|<1$. This path does not lead to identifying insignificant regions for stability. The second problem is a technical one: A main assumption in Doyle and Stein (1981) is that $G_{l}$ and $G_{h}$ have identical residues at the imaginary-axis poles. This assumption is not being made in this paper when we consider case (b) (the multiplicity of the pole at the origin is different in $G_{l}$ than in $G_{h}$ ) so that results from perturbation approach are not directly applicable to case (b). Therefore, to use a perturbation approach even for assessing closed-loop stability, some modification of the standard robustness results would be needed.

\section{Main results}

This section is organized as follows: We first define various quantities that are used in the statements of the main results. In $\S 3.1$, Lemma 1 gives a controller synthesis procedure based on significant poles focusing on closed-loop stability only. In Theorem 1, this procedure is extended to cover both closed-loop stability and performance. Lemma 2 and Theorem 2 in $\S 3.2$ state dual results for insignificant zeros. The case of complex conjugate pairs of polex/zeros is significantly more involves than the real poles/zeros. Corollary 1 in $\$ 3.1$ shows that constraining the candidate insignificant poles to the real-axis results in considerable simplifications.

Let $-\epsilon_{i} \in \mathbb{C}_{-}$and define

$$
\begin{aligned}
a_{i} & :=\operatorname{Re}\left(1 / \epsilon_{i}\right)<0, \quad b_{i}:=\left|\operatorname{Im}\left(1 / \epsilon_{i}\right)\right| \geq 0, \\
m_{i} & :=\left|1 / \epsilon_{i}\right|=\left(a_{i}^{2}+b_{i}^{2}\right)^{1 / 2} .
\end{aligned}
$$

Let $\Delta_{0}:=0$. For $i=1, \ldots, \rho$, define $\Delta_{i} \in \mathbf{S}$ by

$$
\begin{aligned}
1-\Delta_{i} & :=\frac{1}{\epsilon_{i} s+1}, \quad \epsilon_{i} \in \mathbb{R}, \\
1-\Delta_{i} & :=\frac{1}{\left(\epsilon_{i} s+1\right)\left(\bar{\epsilon}_{i} s+1\right)}, \quad \epsilon_{i} \notin \mathbb{R} .
\end{aligned}
$$

Consider $\rho_{1}$ real numbers $\epsilon_{i} \in \mathbb{R}$, and $\rho_{2}=\rho-\rho_{1}$ complex-conjugate pairs $\epsilon_{i}, \bar{\epsilon}_{i} \notin \mathbb{R}$. Let

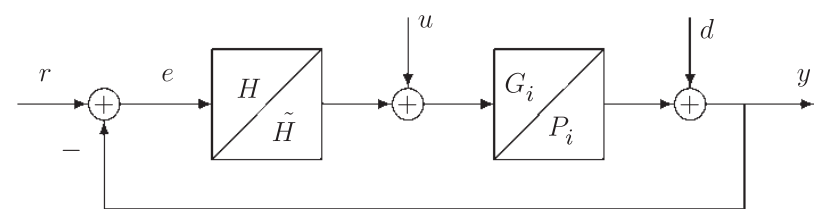

Figure 1. Unity-feedback control system.

It is assumed that the indices $\{1, \ldots, \rho)$ of $\epsilon_{i} \in \mathbb{C}$ are ordered such that $r_{i}>r_{i+1}$. Define

$$
M_{i}=\max \left\{a_{i}, b_{i}\right\}, \quad q_{i}:=\left\|1-\Delta_{i}\right\|=\left(M_{i} / a_{i}+a_{i} / M_{i}\right) / 2 .
$$

Obviously, when $\epsilon_{i} \in \mathbb{R}, b_{i}=0$ implies $M_{i}=a_{i}, q_{i}=1$; otherwise, $q_{i}=1$ when $a_{i} \geq b_{i}$, and $q_{i}=\left(a_{i}^{2}+b_{i}^{2}\right) /$ $2 a_{i} b_{i} \geq 1$ when $b_{i}>a_{i}$. For $k \in\{0, \ldots, \rho-1\}$, and $k+1 \leq i \leq \rho$, define $R_{k i}$ as

$$
R_{k i}:=r_{k+1}+\sum_{j=k+2}^{i} r_{j} \prod_{\ell=k+1}^{j-1} q_{\ell} .
$$

Now let $\tilde{\Delta}_{i}(s):=\Delta_{i}(1 / s)$ so that $\tilde{\Delta}_{0}=0$ and for $i=1, \ldots, \rho, \tilde{\Delta}_{i}$ is defined by

$$
\begin{aligned}
1-\tilde{\Delta}_{i}=1-\Delta_{i}(1 / s) & =\frac{1}{\epsilon_{i} / s+1}=\frac{s}{s+\epsilon_{i}}, \quad \epsilon_{i} \in \mathbb{R}, \\
1-\tilde{\Delta}_{i}=1-\Delta_{i}(1 / s) & =\frac{1}{\left(\epsilon_{i} / s+1\right)\left(\bar{\epsilon}_{i} / s+1\right)} \\
& =\frac{s^{2}}{\left(s+\epsilon_{i}\right)\left(s+\bar{\epsilon}_{i}\right)}, \quad \epsilon_{i} \notin \mathbb{R} .
\end{aligned}
$$

If $\epsilon_{i} \in \mathbb{R}$, then $1-\Delta_{i}(s)$ has a pole at $-1 / \epsilon_{i}$. Under the transformation $s \rightarrow s^{-1}$, the dual term $1 /\left[1-\Delta_{i}(1 / s)\right]=1+\epsilon_{i} / s$ has a zero at $-\epsilon_{i}$. It is easy to see that with $r_{i}, q_{i}$ as in (3) and (4),

$$
\left\|s \tilde{\Delta}_{i}\right\|=\left\|\frac{\Delta_{i}}{S}\right\|=r_{i}, \quad\left\|1-\tilde{\Delta}_{i}\right\|=\left\|1-\Delta_{i}\right\|=q_{i} .
$$

\subsection{Insignificant poles}

Consider the unity-feedback system shown in figure 1 . Let $G \in \mathcal{M}\left(\mathbf{R}_{\mathbf{p}}\right)$ be the plant's transfer matrix, $H \in \mathcal{M}\left(\mathbf{R}_{\mathbf{p}}\right)$ be the controller's transfer matrix. Let $G=N D^{-1}$ be a right-coprime-factorization (RCF), $H=D_{c}^{-1} N_{c}$ be a left-coprime-factorization (LCF) over S. Let $\Delta_{i}$ be defined as in (2). For $i=1, \ldots, \rho$, suppose that $\prod_{j=1}^{i}\left(1-\Delta_{j}\right) I$ is a multiplicative perturbation on the plant $G$. Define $G_{0}:=\left(1-\Delta_{0}\right) G=G$,

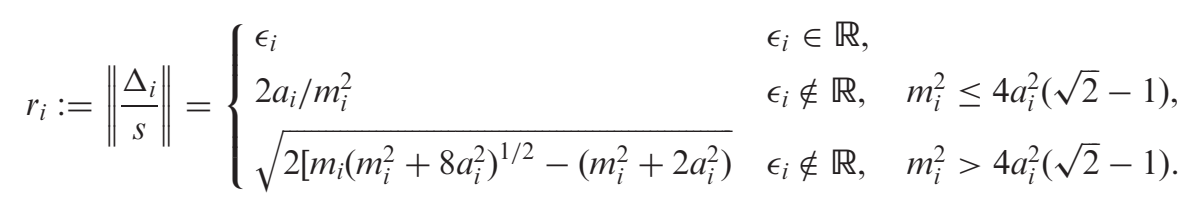


$N_{0}:=\left(1-\Delta_{0}\right) N=N$, and $G_{i}, N_{i}$ as

$$
\begin{aligned}
& G_{i}:=\left(1-\Delta_{i}\right) G_{i-1}=G \prod_{j=1}^{i}\left(1-\Delta_{j}\right), \\
& N_{i}:=\left(1-\Delta_{i}\right) N_{i-1}=N \prod_{j=1}^{i}\left(1-\Delta_{j}\right),
\end{aligned}
$$

where, for $k=0, \ldots, \rho-1, k+1 \leq i \leq \rho$,

$$
\begin{aligned}
\prod_{\ell=k+1}^{i}\left(1-\Delta_{\ell}\right) & =1-\left[\Delta_{k+1}+\sum_{j=k+2}^{i} \Delta_{j} \prod_{\ell=k+1}^{j-1}\left(1-\Delta_{\ell}\right)\right] \\
& =: 1-\Phi_{i} .
\end{aligned}
$$

Clearly, $G_{i}=N_{i} D^{-1}$ is an RCF of $G_{i}$. For $i=1, \ldots, \rho$, with $G_{i}$ as the plant in the unity-feedback system, the sensitivity function $S_{i}$ (i.e., the input-to-error transfer-function) and the complementary sensitivity function $T_{i}=I-S_{i}$ (i.e., the input-to-output transferfunction) are

$$
S_{i}=\left(I+G_{i} H\right)^{-1}, \quad T_{i}=G_{i} H\left(I+G_{i} H\right)^{-1} .
$$

We start formal statement of the results with Lemma 1, which in its simplest form states that if $H$ stabilizes a plant $G$ and if $-1 / \epsilon<-\left\|s G H(1+G H)^{-1}\right\|$, then $H$ also stabilizes the higher order plant $G /(\epsilon S+1)$. In other words, if the plant to be stabilized is $G /(\epsilon S+1)$, then the controller $H$ designed to stabilize the lower order plant $G$ also works for the original plant. The insignificant pole at $s=-1 / \epsilon$ need not be known explicitly; any pole satisfying the norm bound can be in the higher order model. A similar conclusion was stated in Smith and Sondergeld (1986) but only for scalar plants with stable controllers; it was also independently used in Gündeş and Kabuli (2001) to establish a simultaneous stabilization result. This lemma can also be proved as a corollary to the result in Doyle and Stein (1981). In Lemma 1, it is assumed that $G_{k} H$ is strictly-proper, equivalently $T_{k}=G_{k} H\left(I+G_{k} H\right)^{-1} \in \mathcal{M}\left(\mathbf{R}_{\mathbf{s}}\right), S_{k}(\infty)=I$. For $k \geq 1$, $1-\Delta_{k} \in \mathbf{R}_{\mathbf{s}}$ implies $G_{k} H=\left(1-\Delta_{k}\right) G_{k-1} H \in \mathcal{M}\left(\mathbf{R}_{\mathbf{s}}\right)$; hence this assumption is automatically satisfied. For $k=0, G H \in \mathcal{M}\left(\mathbf{R}_{\mathbf{s}}\right)$ if $G \in \mathcal{M}\left(\mathbf{R}_{\mathbf{s}}\right)$ or $H \in \mathcal{M}\left(\mathbf{R}_{\mathbf{s}}\right)$. Any controller $H=D_{c}^{-1} N_{c}$ stabilizing $G=N D^{-1}$ can be modified easily to make it strictly-proper using

$$
H=\left[\left(I+B N_{c} N\right) D_{c}\right]^{-1}\left(I-B D_{c} D\right) N_{c},
$$

where $B:=\left(D_{c} D\right)(\infty)^{-1}$. Therefore, there is no loss of generality in assuming $G_{k} H \in \mathcal{M}\left(\mathbf{R}_{\mathbf{s}}\right)$, with the controller chosen strictly-proper as necessary.

Lemma 1: Suppose that $H$ is a stabilizing controller for the plant $G_{k}$ for some $k \in\{0, \ldots, \rho-1\}$, where $G_{k} H \in \mathcal{M}\left(\mathbf{R}_{\mathbf{s}}\right)$. If

$$
r_{i}<\left\|s T_{i-1}\right\|^{-1}, \quad \text { for } i \geq k+1,
$$

then the same $H$ stabilizes the higher order plants $G_{i}=\left(1-\Delta_{i}\right) G_{i-1}=\prod_{\ell=k+1}^{i}\left(1-\Delta_{\ell}\right) G_{k}$.

Lemma 1 justifies and generalizes to the MIMO case methods in which a stabilizing controller is determined by neglecting the insignificant poles in a loop-gain transfer function and performing the design on the lower order approximation $G$. The terms that are discarded are such that the low-frequency gains $G(0)$ and $G_{k}(0)$ in (8) are the same. Based on condition (12), a real pole at $-1 / \epsilon_{i}$ of (8) is insignificant if $-1 / \epsilon_{i}<-\left\|s T_{i-1}\right\|$, i.e., if it is sufficiently far from the origin in the left-half complex plane. A complex-conjugate pair of poles $\epsilon_{i}, \bar{\epsilon}_{i}$ that has $-1 / r_{i}$ to the left of the line at $\left\|s T_{i-1}\right\|$, would be guaranteed as insignificant. The condition (12) (as well as the condition (13) in Theorem 1 below) requires a "high-frequency performance" from $H$ (see Remark 1 below). This is reasonable to expect since if a controller is highly robust at high frequencies, then it can also tolerate as high a "disturbance" as the introduction of an extra pole at those frequencies to the plant. The definition of an insignificant pole obviously depends on the controller choice due to dependence of $\left\|s T_{i-1}\right\|$ 's on the controller $H$. Theorem 1 incorporates closed-loop performance to Lemma 1.

Theorem 1: Let $H$ be a stabilizing controller for the plant $G_{k}$ for some $k \in\{0, \ldots, \rho-1\}$, where $G_{k} H \in \mathcal{M}\left(\mathbf{R}_{\mathbf{s}}\right)$. For $k+1 \leq i \leq \rho$, let $R_{k i}$ be as in (5) and $\alpha_{i}:=\left\|s T_{i-1}\right\|$. If $R_{k \rho}<\left\|s T_{k}\right\|^{-1}$, i.e., if

$$
\left\|s T_{k}\right\|=\left(R_{k \rho}+\delta\right)^{-1}
$$

for some $\delta>0$, then for $k+1 \leq i \leq \rho$, the same controller $H$ also stabilizes $G_{i}=\left(1-\Delta_{i}\right) G_{i-1}=\Pi_{\ell=k+1}^{i}\left(1-\Delta_{\ell}\right) G_{k}$. Furthermore, $\left\|s T_{i}\right\|$ satisfies

$$
\left\|s T_{i}\right\| \leq\left(R_{i \rho}+\delta \prod_{\ell=k+1}^{i} q_{\ell}^{-1}\right)^{-1},
$$

and the following sensitivity and complementary sensitivity bounds are achieved:

$$
\begin{aligned}
\left(1+r_{i} \alpha_{i}\right)^{-1}\left\|S_{i-1}\right\| & \leq\left\|S_{i}\right\| \leq\left(1-r_{i} \alpha_{i}\right)^{-1}\left\|S_{i-1}\right\|, \\
\left(1+R_{k i} \alpha_{k+1}\right)^{-1}\left\|S_{k}\right\| & \leq\left\|S_{i}\right\| \leq\left(1-R_{k i} \alpha_{k+1}\right)^{-1}\left\|S_{k}\right\|,
\end{aligned}
$$

$$
\begin{aligned}
& \left(1+r_{i} \alpha_{i}\right)^{-1}\left(\left\|T_{i-1}\right\|-r_{i} \alpha_{i}\right) \leq\left\|T_{i}\right\| \leq\left(1-r_{i} \alpha_{i}\right)^{-1} \\
& \quad \times \min \left\{\left(\left\|T_{i-1}\right\|+r_{i} \alpha_{i}\right), q_{i}\left\|T_{i-1}\right\|\right\}, \\
& \left(1+R_{k i} \alpha_{k+1}\right)^{-1}\left(\left\|T_{k}\right\|-R_{k i} \alpha_{k+1}\right) \leq\left\|T_{i}\right\| \leq\left(1-R_{k i} \alpha_{k+1}\right)^{-1} \\
& \quad \times \min \left\{\left\|T_{k}\right\|+R_{k i} \alpha_{k+1},\left\|T_{k}\right\| \prod_{\ell=k+1}^{i} q_{\ell}\right\} .
\end{aligned}
$$


Condition (13) simplifies considerably when all candidate insignificant poles are real.

Corollary 1: Suppose that all $\epsilon_{i} \in \mathbb{R}$ for $k+1 \leq i \leq \rho$. Under the assumptions of Theorem 1 , if there exists a real $\delta>0$ such that

$$
\left\|s T_{k}\right\|=\left(\sum_{j=k+1}^{\rho} \epsilon_{j}+\delta\right)^{-1},
$$

then the same controller $H$ also stabilizes $G_{i}, k+1 \leq i \leq \rho$, and satisfies $\left\|s T_{i}\right\| \leq\left(\sum_{j=i+1}^{\rho} \epsilon_{j}+\delta\right)^{-1}$.

Remark 1: Condition (13) is a high-frequency performance requirement on the plant $G_{k}$. In the scalar case, this condition is equivalent to $\sup _{\omega \geq 0}|\omega| \times$ $\left|T_{k}(j \omega)\right| \leq\left(\delta+R_{k \rho}\right)^{-1}, \quad$ which implies $\quad\left|T_{k}(j \omega)\right| \leq$ $\left(\omega\left(\delta+R_{k \rho}\right)\right)^{-1}$ for all $\omega \geq 0$. This means in particular that $\left|T_{k}(j \omega)\right|<1$ for all $\omega \geq R_{k \rho}^{-1}$. By Theorem 1, a similar performance holds true for each plant $G_{i}$, $i \in[k+1, \rho]$ stabilized by the same controller. If $G_{i}$ has a pole in the open-right-half plane and its associated complementary sensitivity function $T_{i}$ has small magnitude over some frequency range, then its $H_{\infty}$-norm must necessarily get large (Francis and Zames 1984, § V). The bounds in (16) show that $\left\|T_{i}\right\|$ nevertheless remains bounded by a multiple of $\left\|T_{k}\right\|$ and $\left\|s T_{k}\right\|$ (similar comments apply to $\left.\left.\left\|S_{i}\right\|\right)\right)$. In the MIMO case, (13) implies $\bar{\sigma}\left(T_{k}(j \omega)\right) \leq\left(\omega\left(\delta+R_{k \rho}\right)\right)^{-1}$ for all $\omega \geq 0$.

Remark 2: The high-frequency requirement (13) can be represented in terms of the plant $G_{k}$ and any nominal stabilizing controller $H_{o}$ for $G_{k}$. For any LCF $H_{o}=D_{c o}^{-1} N_{c o}, \quad H_{o}$ stabilizes $G_{k}$ if and only if $U_{k}=D_{c o} D+N_{c o} N_{k}$ is unimodular. Let $G_{k}=\tilde{D}_{k}^{-1} \tilde{N}_{k}$ be any LCF of $G_{k}$. All stabilizing controllers for $G_{k}$ are expressed as $\left(D_{c o}-Q \tilde{N}_{k}\right)^{-1}\left(N_{c o}-Q \tilde{D}_{k}\right)$, where $Q \in \mathcal{M}(\mathbf{S})$. Suppose that for some $\delta>0$, $\min _{Q}\left\|s N_{k} U_{k}^{-1}\left(N_{c o}-Q \tilde{D}_{k}\right)\right\|=\left(\delta+R_{k \rho}\right)^{-1}$; the minimum is taken over all $Q \in \mathcal{M}(\mathbf{S})$ such that $N_{k} U_{k}^{-1}\left(N_{c o}-Q \tilde{D}_{k}\right)$ is strictly-proper. If $Q_{*}$ denotes the argument minimum of $\left\|s N_{k} U_{k}^{-1}\left(N_{c o}-Q \tilde{D}_{k}\right)\right\|$, then the controller $D_{c}^{-1} N_{c}:=\left(D_{c o}-Q_{*} \tilde{N}_{k}\right)^{-1}\left(N_{c o}-Q_{*} \tilde{D}_{k}\right)$ satisfies $D_{c} D+N_{c} N_{k}=U_{k}$ and $\left\|s N_{k} U_{k}^{-1} N_{c}\right\|=\left(\delta+R_{k \rho}\right)^{-1}$. Thus (13) holds if and only if $\min _{Q} \| s N_{k} U_{k}^{-1}$ $\left(N_{c o}-Q \tilde{D}_{k}\right) \|<R_{k \rho}^{-1}$, which is a well-known $H_{\infty}$-problem (Francis 1987, Doyle et al. 1989).

Remark 3: Using the consequence (14) of (13), we have $\alpha_{i} \leq\left(\delta+R_{k \rho}\right)^{-1}$ for $i \in[1, \rho]$. Conditions (14) hence remain valid when $\alpha_{i}$ is replaced by $\left(\delta+R_{k \rho}\right)^{-1}$ everywhere it occurs. This gives sensitivity and complementary sensitivity bounds in terms of insignificant poles and the positive constant $\delta$. The resulting bounds, however, are looser than the bounds in terms of $\alpha_{i}$.
Remark 4: Theorem 1 provides an iterative reduction procedure, which normally starts out without any of the left-half plane poles $\left\{-1 / \epsilon_{i}, i=1, \ldots, \rho\right\}$ and checks if (13) can be satisfied by a stabilizing controller for $G$. If not, then the pole(s) $-1 / \epsilon_{i}$ are appended to $G$, staring with the one "closest" to the imaginary-axis. In the case of real poles, if $\epsilon_{i}<\epsilon_{j}$ for some $i, j \in[1, \rho]$, then the pole $-1 / \epsilon_{j}$ is closer to the imaginary-axis, i.e., $-1 / \epsilon_{j}>-1 / \epsilon_{i}$. When all candidate insignificant poles are real (and hence $q_{\ell}=1$ ), we can easily explain why it is reasonable to start the reduction algorithm by appending the right-most real pole to increase the order: Consider two possibilities, $G_{1}^{\ell}=\left(1-\Delta_{1}^{\ell}\right) G, \quad G_{1}^{m}=\left(1-\Delta_{1}^{m}\right) G$, with $\quad \epsilon_{1}^{\ell}>\epsilon_{1}^{m}$. Since $\quad\left(\delta+\epsilon_{1}^{\ell}+\sum_{j=2}^{\rho} r_{j}\right)^{-1} \leq$ $\left(\delta+\epsilon_{1}^{m}+\sum_{j=2}^{\rho} r_{j}\right)^{-1}$, the upper-bound in (13) on $\left\|s T_{1}^{\ell}\right\|$ is larger than the one on $\left\|s T_{1}^{m}\right\|$ (for a controller that achieves similar values for these norms); i.e., for $G_{1}^{\ell}$ and $G_{1}^{m}$ having similar high frequency performances, (13) is easier to satisfy with $G_{1}^{\ell}$ than with $G_{1}^{m}$. Although this simple justification explains why we increase the order by including the right-most real pole, we cannot state a similar easy rule in the case of complex-conjugate pairs of condidate insignificant poles since $q_{\ell} \geq 1$ and the imaginary parts also affect (13).

Remark 5: Based on (17), a real pole at $-1 / \epsilon_{i}$ to the left of the line at $-\left\|s T_{k}\right\|$ can be considered insignificant for order reduction. As $\left\|s T_{k}\right\|$ gets smaller, this line at $-\alpha_{k+1}$ moves closer to the imaginary-axis, enlarging the region for insignificant poles.

Example 1: Consider the single-input single-output plant

$$
G_{2}=\frac{g(s+z)}{\left(\epsilon_{1} s+1\right)\left(\epsilon_{2} s+1\right)(s-p)}=\frac{1}{\left(\epsilon_{1} s+1\right)\left(\epsilon_{2} s+1\right)} G_{o}
$$

with $g, z, p \in \mathbb{R}, z>0$. Let $c \in \mathbb{R}$ be such that $c>-p$. A coprime-factorization of $G=G_{o}$ is $G=N D^{-1}=$ $(g(s+z) /(s+p+c))((s+p) /(s+p+c))^{-1}$. Then $H=$ $(c / g(s+z))$ is a stabilizing controller for $G$, and $G H$ is strictly-proper. From (10), $T_{0}=(c /(s+p+c))$, $S_{0}=I-T_{0}=D$, and $\alpha_{1}:=\left\|s T_{0}\right\|=c$. By Theorem 1 , there exists $\delta>0$ satisfying (13) for $k=0$ if and only if $c<R_{o \rho}^{-1}$. Obviously, it is possible to choose $c \in \mathbb{R}$ to satisfy this constraint for any set of insignificant poles provided $-p<R_{0 \rho}^{-1}$.

(a) First consider two real candidate insignificant poles at $-1 / \epsilon_{2}<-1 / \epsilon_{1}, \epsilon_{1}=5, \epsilon_{2}=1$. Suppose $-p<1 / 6$. If we choose $c=1 / 8<1 /\left(\epsilon_{1}+\epsilon_{2}\right)$, then by (13), $\delta=2$. By Theorem 1, the controller $H=(0.125 /$ $g(s+z)$ that was designed to stabilize the lower order system $G=G_{o}$ also stabilizes the higher order plant $\left.G_{1}=G_{o} /(5 s+1)\right)$ and the original plant $G_{2}=G_{1} /(s+1)$. 
(b) Instead of two real poles, now consider a complex-conjugate pair of insignificant poles at $-1 / \epsilon_{1}, \quad-1 / \bar{\epsilon}_{1}$. First let $1 / \epsilon_{1}=0.2+j 0.15$, i.e., $\epsilon_{1}=3.2-j 2.4, r_{1}=6.4, q_{1}=1$, as in (3) and (4). If we choose $c=1 / 8<1 / r_{1}$, then $\delta=1.6$. By (13), the same controller $H$ also stabilizes the original higher order plant $G_{2}=G /\left(16 s^{2}+6.4 s+1\right)$. For a different choice, let $1 / \epsilon_{1}=0.16+j 1$, i.e., $\epsilon_{1}=0.156+j 0.975$, $r_{1}=3.2775, q_{1}=3.205$. If we choose $c=1 / 8<1 / r_{1}$, then $\delta=4.7225$. By (13), the same controller $H$ also stabilizes the original higher order plant $G_{2}=G_{o} /\left(0.975 s^{2}+0.312 s+1\right)$.

We now verify the bounds in (14)-(16) by tabulating the norms of the sensitivity function in (18)-(19). For this purpose we used the two different values $p=0$ and $p=0.1$ for the plant pole at $s=p$

Example 2: Consider a single-input single-output, also explore two other full-order observer-based controllers and the corresponding guaranteed region for insignificant poles: A state-space representation $(A, B, C, D)$ for $G_{o}$ is given by

$$
\begin{aligned}
A & =\left[\begin{array}{ccc}
0.25 & -3.375 & 0.84375 \\
1 & 0 & 0 \\
0 & 1 & 0
\end{array}\right], \\
B & =\left[\begin{array}{lll}
1 & 0 & 0
\end{array}\right]^{T}, \quad C=\left[\begin{array}{lll}
1 & 0 & 0.5625
\end{array}\right], \quad D=0 .
\end{aligned}
$$

We place the eigenvalues of $(A-B F)$ at $\{-0.6,-0.7$, $-0.8\}$ and the eigenvalues of $(A-L C)$ at $\{-1,-0.8$, $\pm j 0.2\} \quad$ using $F=\left[\begin{array}{lll}2.35 & -1.915 & 1.1797\end{array}\right]$ and $\left.L^{T}=2.963 \quad 0.3391-0.2009\right]$. For the third-order stable controller

\begin{tabular}{|c|c|c|c|c|}
\hline \multicolumn{3}{|c|}{$\epsilon_{1}=3.2-j 2.4$} & \multicolumn{2}{|c|}{$\epsilon_{1}=0.156-j 0.975$} \\
\hline & $p=-1 / 10$ & & $p=0$ & $p=-0.1$ \\
\hline$\left\|s T_{0}\right\|,\left\|T_{0}\right\|,\left\|S_{0}\right\|$ & $c, 1,1$ & $c, 5,4$ & $c, 1,1$ & $c, 5,4$ \\
\hline$\left\|s T_{1}\right\|,\left\|T_{1}\right\|,\left\|S_{1}\right\|$ & $0.196,1.302,1.8888$ & $0.4676,6.377,6.3398$ & $0.6652,1,1.6609$ & $0.6674,5$ \\
\hline
\end{tabular}

\begin{tabular}{l|l|l}
\multicolumn{3}{|c|}{$\epsilon_{1}=5, \quad \epsilon_{2}=1$} \\
\hline$\left\|s T_{0}\right\|,\left\|T_{0}\right\|,\left\|S_{0}\right\|$ & $c, 1,1$ & $p=-0.1$ \\
$\left\|s T_{1}\right\|,\left\|T_{1}\right\|,\left\|S_{1}\right\|$ & $c, 1.0193,1.3247$ & $c, 5,4$ \\
$\left\|s T_{2}\right\|,\left\|T_{2}\right\|,\left\|S_{2}\right\|$ & $0.1407,1.0862,1.4813$ & $0.25,5,4.1798$ \\
\end{tabular}

unstable, non-minimum phase, strictly-proper plant

$$
G_{i}=\frac{32 s^{2}+18}{(4 s-1)\left(8 s^{2}+27\right)} \prod_{i=1}^{\rho} \frac{1}{\left(\epsilon_{i} s+1\right)}=G_{o} \prod_{i=1}^{\rho} \frac{1}{\left(\epsilon_{i} s+1\right)}
$$

where $G=G_{o}$ has poles on the imaginary-axis. Clearly, $G_{o}$ is stabilized by any constant controller $H_{1}>1.5$. If we choose $H_{1}=3.37$, then $\alpha_{1}=\left\|s T_{0}\right\|=3.37$, with closed-loop poles at $\{-0.5199,-1.300 \pm j 0.5777\}$. By Theorem 1, (13) is holds for $k=0$ if and only if $R_{0 \rho}<1 /$ $\alpha_{1}=0.2967$. For example, a single pole at $-1 / \epsilon_{1}<-3.37$ is guaranteed to be insignificant; if $\epsilon_{1}=0.25$, then the controller $H_{1}=3.37$ also stabilizes the higher order plant $G_{1}=G_{o} /(0.25 s+1)$ (equivalently, the controller $4 H_{1}$ stabilizes $G_{o} /(s+4)$ ), with closed-loop poles at $\{-0.4528 \pm j 0.3460,-1.4222 \pm j 3.3063\}$. We 
$F_{3}=\left[\begin{array}{lll}0.8833 & 0.1193 & 1.7448\end{array}\right]$; using $Q=50 B B^{T}, R=1$, we

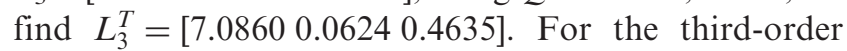
stable controller

$$
\begin{aligned}
H_{3}: & =F_{3}\left(s I-A-B F_{3}+L_{3} C\right)^{-1} L_{3} \\
& =\frac{7.0753 s^{2}+0.9096 s+15.1593}{s^{3}+7.98 s^{2}+3.0589 s+5.7641},
\end{aligned}
$$

we obtain $\alpha_{1}=\left\|s T_{0}\right\|=2.0138$, with closed-loop poles at $\{-6.6589,-0.2653,-0.1840 \pm j 1.8338, \quad-0.2189 \pm$ $j 0.7501\}$. Using $H_{3}$, any number of insignificant poles can be added to $G$ provided that $R_{0 \rho}<1 / \alpha_{1}=0.4966$.

\section{Example 3:}

(a) Consider an MIMO plant represented by its transfer-function

Consider the lower order system $G_{o}$ in (20). An $\mathrm{RCF}$ of

$$
\begin{aligned}
G_{3} & =\prod_{i=1}^{3} \frac{1}{\left(\epsilon_{i} s+1\right)}\left[\begin{array}{c}
\frac{(s+1)(s+6)(s-4)}{(5 s+8)\left(s^{2}+40\right)} \\
\frac{-(s+1)(s+6)(s-4)}{(s+2)(5 s+8)\left(s^{2}+40\right)}
\end{array}\right. \\
& =\frac{1}{(0.03 s+1)(0.02 s+1)(0.01 s+1)} G_{o} .
\end{aligned}
$$

$$
\begin{aligned}
G= & G_{o} \text { is } G=N D^{-1} \\
& =\left[\begin{array}{ccc}
\frac{(s-4)}{(5 s+8)} & \frac{s}{(2 s+3)} & 0 \\
\frac{-(s-4)}{(s+2)(5 s+8)} & \frac{s(s+1)}{(s+2)(2 s+3)} & 0
\end{array}\right] \\
& \times\left[\begin{array}{ccc}
\frac{s^{2}+40}{(s+1)(s+6)} & 0 & 0 \\
0 & \frac{s-3}{s+6} & \frac{-(s-1)}{s+6} \\
0 & 0 & 1
\end{array}\right] .
\end{aligned}
$$

A stabilizing controller for $G_{o}$ is

$$
\begin{aligned}
H= & D_{c}^{-1} N_{c}=\left[\begin{array}{ccc}
\frac{5(s+1)}{5 s+8} & 0 & 0 \\
0 & \frac{2(s-3)}{2 s+3} & \frac{2(s-3)(s-1)}{(2 s+3)(s+6)} \\
0 & 0 & 1
\end{array}\right]^{-1} \\
& \times\left[\begin{array}{cc}
\frac{38(s+1)}{(s+2)(s+6)} & \frac{-38}{s+6} \\
\frac{27}{(s+2)(s+6)} & \frac{27}{s+6} \\
0 & 0
\end{array}\right] .
\end{aligned}
$$

From (10), $T_{0}=N N_{c}$, and $\alpha_{1}:=\left\|s T_{0}\right\|=15.1266$. By Theorem 1, there exists $\delta>0$ satisfying (13) for $k=0$ if and only if $\alpha_{1}<R_{03}^{-1}$. With $\epsilon_{1}=0.03$, $\epsilon_{2}=0.02, \quad \epsilon_{3}=0.01, \quad$ we have $\delta=\alpha_{1}^{-1}-$ $\sum_{j=1}^{3} \epsilon_{j}=0.0061>0$. Therefore, the controller $H$ also stabilizes the higher order plants $G_{i}=G_{i-1} /\left(\epsilon_{i} s+1\right)$, i.e., $\quad G_{1}=G_{o} /(0.03 s+1) \quad$ and $G_{2}=G_{1} /(0.02 s+1)=G_{o} /(0.03 s+1)(0.02 s+1)$ and the original plant $G_{3}$ in (20).

(b) Now consider the MIMO plant

$$
G_{2}=\frac{2125}{\left(s^{2}+70 s+2125\right)(0.025 s+1)} G_{o},
$$

where $G_{o}$ is the same as in (20). The candidate insignificant poles are at $-1 / \epsilon_{1}=-(35+j 30),-1 / \bar{\epsilon}_{1}$ and $-1 / \epsilon_{2}=-40$. With $a_{1}>b_{1}, q_{1}=1, r_{1}=0.0329$, $r_{2}=0.025<r_{1}$, we have $\delta=\alpha_{1}^{-1}-R_{03}=\alpha_{1}^{-1}-$ $\left(r_{1}+r_{2} q_{1}\right)=0.0082>0$. By Theorem 1, the controller $H$ in (21) stabilizes the higher order plants $\quad G_{1}=G /\left(\epsilon_{1} s+1\right)\left(\tilde{\epsilon}_{1} s+1\right)=\left(2125 /\left(s^{2}+\right.\right.$

$$
\left.\begin{array}{cc}
\frac{s(s+6)}{(2 s+3)(s-3)} & \frac{s(s-1)}{(2 s+3)(s-3)} \\
\frac{s(s+1)(s+6)}{(s+2)(2 s+3)(s-3)} & \frac{s\left(s^{2}-1\right)}{(s+2)(2 s+3)(s-3)}
\end{array}\right]
$$$$
\begin{aligned}
& G_{1} /(0.025 s+1) \\
& G_{0}
\end{aligned}
$$

(c) Now consider the MIMO plant

$$
G_{2}=\frac{2125}{\left(s^{2}+60 s+2125\right)(0.025 s+1)} G_{o}
$$

where $G_{o}$ is the same as in (20). This time $-1 / \epsilon_{1}=-(30+j 35), \quad$ with $a_{1}<b_{1} . \quad$ Then $q_{1}=1.0119, \quad r_{1}=0.0308, \quad r_{2}=0.025<r_{1}, \quad \delta=$ $\alpha_{1}^{-1}-R_{03}=0.01>0$. Again, by Theorem 1, the controller $H$ in (21) stabilizes the higher order plants $G_{1}=G /\left(\epsilon_{1} s+1\right)\left(\bar{\epsilon}_{1} s+1\right)=$ $\left(2125 /\left(s^{2}+60 s+2125\right)\right) G_{o}$ and the original plant $G_{2}=G_{1} /(0.025 s+1)$.

\subsection{Insignificant zeros}

Consider the unity-feedback system again. Let $P \in \mathcal{M}\left(\mathbf{R}_{\mathbf{p}}\right)$ be the plant's transfer matrix, $\tilde{H} \in \mathcal{M}\left(\mathbf{R}_{\mathbf{p}}\right)$ be the controller's transfer matrix. Let $P=\tilde{D}^{-1} \tilde{N}$ be an $\mathrm{LCF}, \tilde{H}=\tilde{N}_{c} \tilde{D}_{c}^{-1}$ be and RCF over S. Let $P$ be full row-rank and have no transmission-zeros at $s=0$, equivalently, let $\tilde{N}(0)$ be full row-rank.

Let $\tilde{\Delta}_{i}$ be defined as in (6). For $i=1, \ldots, \rho$, suppose that $\Pi_{j=1}^{i}\left(1-\tilde{\Delta}_{j}\right)^{-1} I$ is a multiplicative perturbation on the plant $P$. Define $P_{0}:=\left(1-\tilde{\Delta}_{0}\right)^{-1} P=P$, $\tilde{D}_{0}:=\left(1-\tilde{\Delta}_{0}\right) \tilde{D}=\tilde{D}$, and $P_{i}, \tilde{D}_{i}$ as 


$$
\begin{aligned}
& P_{i}:=\left(1-\tilde{\Delta}_{i}\right)^{-1} P_{i-1}=P \prod_{j=1}^{i}\left(1-\tilde{\Delta}_{j}\right)^{-1}, \\
& \tilde{D}_{i}:=\left(1-\tilde{\Delta}_{i}\right) \tilde{D}_{i-1}=\tilde{D} \prod_{j=1}^{i}\left(1-\tilde{\Delta}_{j}\right),
\end{aligned}
$$

where, for $k=0, \ldots, \rho-1, k+1 \leq i \leq \rho$,

$$
\begin{aligned}
\prod_{\ell=k+1}^{i}\left(1-\tilde{\Delta}_{\ell}\right) & =1-\left[\tilde{\Delta}_{k+1}+\sum_{j=k+2}^{i} \tilde{\Delta}_{j} \prod_{\ell=k+1}^{j-1}\left(1-\tilde{\Delta}_{\ell}\right)\right] \\
& =: 1-\tilde{\Phi}_{i} .
\end{aligned}
$$

Clearly, $P_{i}=\tilde{D}_{i}^{-1} \tilde{N}_{i}$ is an LCF of $P_{i}$. For $i=1, \ldots, \rho$, with $P_{i}$ as the plant in the unity-feedback control system, the sensitivity function $S_{i}$ and the complementary sensitivity function $T_{i}=I-S_{i}$ are given by (10), with $P_{i}, \tilde{H}$ replacing $G_{i}, H$.

In $\S 3.1,1-\Delta_{i}(s)$ has a pole at $-1 / \epsilon_{i}$ (or a complexconjugate pair of poles at $\left.-1 / \epsilon_{i},-1 / \bar{\epsilon}_{i}\right)$; here, $1-\Delta_{i}(1 / s)=1-\tilde{\Delta}_{i}(s)$ has a zero at $-\epsilon_{i}$ (or a complexconjugate pair of zeros at $\left.-\epsilon_{i},-\bar{\epsilon}_{i}\right)$. Therefore, $P$ can be considered as a reduced order plant obtained from the higher order plant $P_{\rho}=\Pi_{j=1}^{\rho}\left(1-\tilde{\Delta}_{j}\right)^{-1} P$ by canceling zeros in the stable region with poles at the origin. The order of $P_{\rho}$ is $\rho_{1}+2 \rho_{2}$ more than the of $P$; the additional $\rho_{1}$ (negative) real zeros at $-\epsilon_{i}$ and the $\rho_{2}=\rho-\rho_{1}$ pairs of complex-conjugate zeros at $-\epsilon_{i}, \bar{\epsilon}_{i}$ of $P_{\rho}$ are called candidate insignificant zeros; $P_{\rho}$ has $\rho$ additional poles at $s=0$. It is clear that the insignificant poles represented by the perturbation $1-\Delta_{i}(s)$ in $\S 3.1$ and the insignificant zeros represented by the perturbation $\left(1-\Delta_{i}(1 / s)\right)^{-1}$ in this section are dual concepts. The equality of the norms $\left\|\Delta_{i} / s\right\|=\left\|s \Delta_{i}(1 / s)\right\|$ and $\left\|1-\Delta_{\mathrm{i}}(s)\right\|=\left\|1-\Delta_{i}(1 / s)\right\|$ as stated in (7) help to establish similar results for insignificant zeros through the transformation $s \rightarrow s^{-1}$.

In $\S 3.1$, where $1-\Delta_{k}(\infty)=0$, it was assumed that $G_{k} H$ is strictly-proper, equivalently $T_{k}(\infty)=0$, $S_{k}(\infty)=I$. In the dual results of this section, where $1-\tilde{\Delta}_{k}(0)=0$, it is assumed that $S_{k}(0)=0=I-T_{k}(0)$, which implies $P_{k} \tilde{H}$ has poles at $s=0$. We say that the transfer matrix $P_{k} \tilde{H}$ is of (type-1 or greater) iff $S_{k}(0)=0$. For $k \geq 1, P_{k} \tilde{H}$ automatically has poles at $s=0$ since $\tilde{D}_{k}(0)=\left(1-\tilde{\Delta}_{k}(0)\right) \tilde{D}_{k-1}(0)$. For $k=0$, this assumption is satisfied if $P=\tilde{D}^{-1} \tilde{N}$ is such that $\tilde{D}(0)=0$ or if $\tilde{H}=\tilde{N}_{c} \tilde{D}_{c}^{-1}$ is such that $\tilde{D}_{c}(0)=0$, in which case we say that the stabilizing controller has integral-action. Any controller $\tilde{H}=\tilde{N}_{c} \tilde{D}_{c}^{-1}$ stabilizing $P=\tilde{D}^{-1} \tilde{N}$ can be one with integral action using a simple modification as

$$
\tilde{H}=\tilde{N}_{c}\left(I+\tilde{D} \tilde{D}_{c} \tilde{B}\right)\left[\tilde{D}_{c}\left(I-\tilde{N} \tilde{N}_{c} \tilde{B}\right)\right]^{-1},
$$

where $\tilde{B}=\left(\tilde{N} \tilde{N}_{c}\right)(0)^{-1}$. Therefore, there is no loss of generality in assuming $S_{k}(0)=0$, with the controller chosen to have integral action as necessary. We now present a dual of Lemma 1.

Lemma 2: Suppose that $\tilde{H}$ is a stabilizing controller for the plant $P_{k}$ for some $k \in\{0, \ldots, \rho-1\}$, where $P_{k} \tilde{H}$ is of type-1 or greater. If

$$
r_{i}<\left\|s^{-1} S_{i-1}\right\|^{-1}, \quad \text { for } i \geq k+1,
$$

then the same $\tilde{H}$ stabilizes the higher order plants $P_{i}:=\left(1-\tilde{\Delta}_{i}\right)^{-1} P_{i-1}=\Pi_{\ell=k+1}^{i}\left(1-\tilde{\Delta}_{\ell}\right)^{-1} P_{k}$.

Lemma 2 justifies methods of stabilizing controller design where a loop-gain transfer function is approximated by a function which is of type- 1 or greater. The terms that are discarded are such that the highfrequency gain of $P$ and that of $P_{k}$ in (22) are the same, i.e., each insignificant zero is cancelled with exactly one pole at the origin. A real zero $-\epsilon_{i}$ is insignificant, or can be discarded together with a pole at the origin, if $-\epsilon_{i}$ is in the interval $\left(-1 / \beta_{i}, 0\right)$, where $\beta_{i}:=\left\|s^{-1} S_{i-1}\right\|$, i.e., it is sufficiently close to the origin. Based on condition (25), a complex-conjugate pair of zeros are cancellable with two poles at the origin if the associated $r_{i}<1 / \beta_{i}$. We now present a dual of Theorem 1. If for some $k<\rho$, we can determine a stabilizing controller that achieves a certain closed-loop performance as measured by $\left\|S^{-1} S_{k}\right\|$ for $P_{k}$, then the same controller stabilizes every $P_{i}$ for $i \geq k$ and has, to some degree, a guaranteed closed-loop performance.

Theorem 2: Let $\tilde{H}$ be a stabilizing controller for the plant $P_{k}$ for some $k \in\{0, \ldots, \rho-1\}$, where $S_{k}(0)=0$. For $k+1 \leq i \leq \rho$, let $R_{k i}$ be as in (5) and $\beta_{i}:=\left\|s^{-1} S_{i-1}\right\|$. If $R_{k \rho}<s^{-1} S_{k}^{-1}$, i.e., if

$$
\left\|s^{-1} S_{k}\right\|=\left(R_{k \rho}+\tilde{\delta}\right)^{-1}
$$

for some $\tilde{\delta}>0$, then for $k+1 \leq i \leq \rho$, the same controller $\tilde{H}$ also stabilizes $P_{i}=\left(1-\tilde{\Delta}_{i}\right)^{-1} P_{i-1}=$ $\Pi_{\ell=k+1}^{i}\left(1-\tilde{\Delta}_{\ell}\right)^{-1} P_{k}$. Furthermore, $\left\|s^{-1} S_{i}\right\|$ satisfies

$$
\left\|s^{-1} S_{i}\right\| \leq\left(R_{i \rho}+\tilde{\delta} \prod_{\ell=k+1}^{i} q_{\ell}^{-1}\right)^{-1},
$$

and the following sensitivity and complementary sensitivity bounds are achieved:

$$
\begin{aligned}
& \left(1+r_{i} \beta_{i}\right)^{-1}\left(\left\|S_{i-1}\right\|-r_{i} \beta_{i}\right) \leq\left\|S_{i}\right\| \leq\left(1-r_{i} \beta_{i}\right)^{-1} \\
& \quad \times \min \left\{\left(\left\|S_{i-1}\right\|+r_{i} \beta_{i}\right), q_{i}\left\|S_{i-1}\right\|\right\}, \\
& \left(1+R_{k i}, \beta_{k+1}\right)^{-1}\left(\left\|S_{k}\right\|-R_{k i} \beta_{k+1}\right) \leq\left\|S_{i}\right\| \leq\left(1-R_{k i} \beta_{k+1}\right)^{-1} \\
& \quad \times \min \left\{\left\|S_{k}\right\|+R_{k i} \beta_{k+1},\left\|S_{k}\right\| \prod_{\ell=k+1}^{i} q_{\ell}\right\},
\end{aligned}
$$




$$
\begin{aligned}
& \left(1+r_{i} \beta_{i}\right)^{-1}\left\|T_{i-1}\right\| \leq\left\|T_{i}\right\| \leq\left(1-r_{i} \beta_{i}\right)^{-1}\left\|T_{i-1}\right\|, \\
& \left(1+R_{k i} \beta_{k+1}\right)^{-1}\left\|T_{k}\right\| \leq\left\|T_{i}\right\| \leq\left(1-R_{k i} \beta_{k+1}\right)^{-1}\left\|T_{k}\right\| .
\end{aligned}
$$

Remark 6: Condition (26) is a low-frequency performance requirement on the plant $P_{k}$. In the scalar case, it is equivalent to $\sup _{\omega \geq 0}|\omega|^{-1}\left|S_{k}(j \omega)\right| \leq\left(\tilde{\delta}+R_{k \rho}\right)^{-1}$, which implies $\left|S_{k}(j \omega)\right| \leq|\omega|\left(\delta+R_{k \rho}\right)^{-1}$ for all $\omega \geq 0$. This means in particular that $\left|S_{k}(j \omega)\right|<1$ for all $\omega \leq R_{k \rho}$. By Theorem 2, a similar performance holds true for each plant $P_{k}, i \in[k+1, \rho]$, stabilized by the same controller. Again by Francis and Zames (1984), if $P_{k}$ has a strict right-half plane zero and its associated sensitivity function gets small in magnitude in a frequency range, then its $H_{\infty}$-norm necessarily gets large. The bounds in (28) show that $\left\|S_{i}\right\|$ nevertheless remain bounded by a multiple of $\left\|S_{k}\right\|$ and $\left\|s^{-1} S_{k}\right\|$.

Remark 7: As a dual of Corollary 1, Theorem 2 is easily simplified when all insignificant zeros are real: Let all $\epsilon_{i} \in \mathbb{R}$ for $k+1 \leq i \leq \rho$. If there exists a real $\tilde{\delta}>0$ such that $\left\|s^{-1} S_{k}\right\|=\left(\sum_{j=k+1}^{\rho} \epsilon_{j}+\tilde{\delta}\right)^{-1}$, then $\tilde{H}$ also stabilizes $P_{i}$ and satisfies $\left\|s^{-1} S_{i}\right\|<\left(\sum_{j=i+1}^{\rho} \epsilon_{j}+\tilde{\delta}\right)^{-1}$. A real zero at $-\epsilon_{i}$ is cancellable if $\epsilon_{i}<\left\|s^{-1} S_{k}\right\|^{-1}$, i.e., it lies in a region between the imaginary-axis and the line at $-1 / \beta_{k+1}$. As $\left\|s^{-1} S_{k}\right\|$ gets smaller, this region gets larger.

Remark 8: The low-frequency requirement (26) can be represented in terms of the plant $P_{k}$ and nominal stabilizing controller $\tilde{H}_{o}$ for $P_{k}$. For any RCF $\tilde{H}_{o}=\tilde{N}_{c o} \tilde{D}_{c o}^{-1}, \quad \tilde{H}_{o}$ stabilizes $P_{k}$ if and only if $V_{k}=\tilde{D}_{k} \tilde{D}_{c o}+\tilde{N} \tilde{N}_{c o}$ is unimodular. Let $P_{k}=\hat{N}_{k} \hat{D}_{k}^{-1}$ be
Example 4: Consider the single-input single-output plant

$$
P_{2}=\frac{(s+10)(s+6)}{s^{2}} \frac{g}{(s-p)}=\frac{\left(s+\epsilon_{1}\right)\left(s+\epsilon_{2}\right)}{s^{2}} P_{o}
$$

with $g, p \in \mathbb{R}$. A coprime-factorization of $P=P_{o}$ is $P=\tilde{D}^{-1} \tilde{N}=((s-p) /(s+c))^{-1}(g /(s+c))$ where $c>0$. Clearly, $\tilde{H}=(c+p) / g$ is a stabilizing controller, and if we modify it to have integral action as in (24), then $\tilde{H}=\left((2 c+p) s+c^{2}\right) / g s$. From $\quad(10), \quad S_{0}=(s(s-p) /$ $\left.(s+c)^{2}\right), \quad T_{0}=I-S_{0}=\left(s /(s+c)^{2}\right) \tilde{H}, \quad$ and $\quad \beta_{1}:=$ $\left\|s^{-1} S_{0}\right\| \leq \max \left\{1 / c,|p| / c^{2}\right\}$. By Theorem 2, (26) holds for $k=0$ if and only if $\beta_{1}<R_{0 \rho}^{-1}$. Obviously, it is possible to choose $c>|p|$ in order to satisfy this constraint for any set of insignificant zeros. Suppose $p=8$.

(a) First consider two real candidate insignificant zeros at $-\epsilon_{1}<-\epsilon_{2}$, where $\epsilon_{1}=10, \epsilon_{2}=6$. If we choose $c=20>\epsilon_{1}+\epsilon_{2}$, then $\beta_{1}=0.0273$, and by (26), $\delta=20.675$. The controller $\tilde{H}=((48 s+400) / s)$ also stabilizes the higher order plant $P_{1}=((s+10) / s) P_{o}$, and the original higher order plant $P_{2}=(s+10) /$ $s) P_{0}$, and the original higher order plant $P_{2}=((s+6) / s) P_{1}$.

(b) Instead of these two real zeros, now consider a complex-conjugate pair of insignificant zeros at $-\epsilon_{1}, \quad-\bar{\epsilon}_{1}$, with $\epsilon_{1}=-10+j 5,1 / \epsilon_{1}=0.08+j 0.04$, $r_{1}=20, q_{1}=1$. With $c=20, \tilde{\delta}=16.675$ by (26); the same controller $\tilde{H}$ also stabilizes the original higher order plant $P_{2}=\left(\left(s^{2}+20 s+125\right) / s^{2}\right) P$.

The bounds in (27)-(29) are easily verified from (30) for the real and complex-conjugate zeros considered

$$
\begin{array}{l|c|c} 
& \epsilon_{1}=6, \epsilon_{2}=10 & \epsilon_{1}=10+5 j \\
\hline\left\|s^{-1} S_{0}\right\|,\left\|T_{0}\right\|,\left\|S_{0}\right\| & 0.0273,1.3196,1 & 0.0273,1.3196,1 \\
\left\|s^{-1} S_{1}\right\|,\left\|T_{1}\right\|,\left\|S_{1}\right\| & 0.0299,1.5492,1.0135 & 0.0372,1.9433,1.2757 \\
\left\|s^{-1} S_{2}\right\|,\left\|T_{2}\right\|,\left\|S_{2}\right\| & 0.0337,1.7622,1.1266 &
\end{array}
$$

any RCF of $P_{k}$. All stabilizing controllers for $P_{k}$ are expressed as $\left(\tilde{N}_{c o}+\hat{D}_{k} Q\right)\left(\tilde{D}_{c o}-\hat{N}_{k} Q\right)^{-1}$, where $Q \in \mathcal{M}(\mathbf{S})$. Suppose that for some $\tilde{\delta}>0$, $\min _{Q}\left\|s^{-1}\left(\tilde{D}_{c o}-\hat{D}_{k} Q\right) V_{k}^{-1} \tilde{D}_{k}\right\|=\left(\tilde{\delta}+R_{k \rho}\right)^{-1}$; the minimum is taken over all $Q \in \mathcal{M}(\mathbf{S})$ such that $\left[\left(\tilde{D}_{c o}-\hat{D}_{k} Q\right) V_{k}^{-1} \tilde{D}_{k}\right](0)=0$. If $Q_{*}$ denotes the argument minimum of $\left\|s^{-1}\left(\tilde{D}_{c o}-\hat{D}_{k} Q\right) V_{k}^{-1} \tilde{D}_{k}\right\|$, then the controller $\tilde{N}_{c} \tilde{D}_{c}^{-1}:=\left(\tilde{N}_{c o}+\hat{D}_{k} Q_{*}\right)\left(D_{c o}-\hat{N}_{k} Q_{*}\right)^{-1}$ stabilizes $P_{k}$ and satisfies $\tilde{D}_{k} \tilde{D}_{c}+\tilde{N} \tilde{N}_{c}=V_{k}$ and $\left\|s^{-1} S_{k}\right\|=\left(\tilde{\delta}+R_{k \rho}\right)^{-1}$. Thus (26) holds if and only if $\min _{Q}\left\|s^{-1}\left(\tilde{D}_{c o}-\tilde{D}_{k} Q\right) V_{k}^{-1} \tilde{D}_{k}\right\|<R_{k \rho}^{-1}$, which in turn is again a well-known $H_{\infty}$-problem.

\section{Conclusions}

In Theorem 1 and 2, we provided dual methods of controller design for MIMO systems based on reduced order models from the viewpoint of closed-loop stability and performance. The iterative design algorithm hinge on the existence of a controller having a certain performance as quantified by conditions (13) and (26). The most important merit of the methods presented is that they directly focus on closed-loop performance and provide estimates in terms of eliminated poles or zeros 
for achievable performance and stability robustness. The design methods provide an MIMO generalization of the scalar design approximation methods. It should be noted that the candidate insignificant poles (or zeros) are "blocking" poles (or zeros) in the sense that they appear in every entry of the transfer matrix. These methods do not restrict the approximated plant to be stable or minimum-phase; the only requirement is that the discarded poles (or zeros) are in the open left-half plane. Unlike most other reduction methods, these do not require any additive decomposition of the plant into stable and anti-stable parts.

\section{Acknowledgement}

Research support by the NSF Grant ECS-9905729 was given to A. N. Gündeş

\section{Appendix}

Proof of Lemma 1: Let $G=N D^{-1}$ be an RCF and let $H=D_{c}^{-1} N_{c}$ be an LCF. For $k \geq 0$, the controller $H$ stabilizes $G_{k}$ if and only if $U_{k}:=D_{c} D+N_{c} N_{k}$ is unimodular. By assumption, for some $k \geq 0, U_{k}$ is unimodular since $H$ stabilizes $G_{k}$. We show that $H$ also stabilizes $G_{i}$ by induction. Suppose that $U_{i-1}$ is unimodular, which is already given for $i=k+1$. Then $N_{i}=\left(1-\Delta_{i}\right) N_{i-1}$ implies

$$
\begin{aligned}
U_{i} & =D_{c} D+N_{c} N_{i}=U_{i-1}-N_{c} N_{i-1}+N_{c} N_{i} \\
& =U_{i-1}-\Delta_{i} N_{c} N_{i-1} .
\end{aligned}
$$

By (31), $U_{i}$ is unimodular if and only if $U_{i-1}^{-1} U_{i}=I-U_{i-1}^{-1} \Delta_{i} N_{c} N_{i-1}$ is unimodular, equivalently, $\tilde{U}_{i}:=I-\Delta_{i} N_{i-1} U_{i-1}^{-1} N_{c}=I-\Delta_{i} T_{i-1}$ is unimodular. Since $G_{k} H \in \mathcal{M}\left(\mathbf{R}_{\mathbf{s}}\right)$ implies $G_{i-1} H=\prod_{\ell=k+1}^{i-1}\left(1-\Delta_{\ell}\right) \times$ $G_{k} H \in \mathcal{M}\left(\mathbf{R}_{\mathbf{s}}\right)$, we have $T_{i-1} \in \mathcal{M}\left(\mathbf{R}_{\mathbf{s}}\right)$ for $i \geq k+1$, and consequently, $s T_{i-1} \in \mathcal{M}(\mathbf{S})$. By (2) $\left(\Delta_{i} / s\right) \in \mathbf{S}$. By (3), if condition (12) holds, then $\left\|\left(\Delta_{i} / s\right) s T_{i-1}\right\| \leq$ $\left\|\left(\Delta_{i} / s\right)\right\|\left\|s T_{i-1}\right\|=r_{i}\left\|s T_{i-1}\right\|<1$. Therefore, $\quad \tilde{U}_{i}=I-$ $\left(\Delta_{i} / s\right) s T_{i-1} \quad$ is unimodular, equivalently, $H$ stabilizes $G_{i}$.

Proof of Theorem 1: For $k+1 \leq i \leq \rho, H$ stabilizes $G_{i}$ if and only if $U_{i}$ in (31) is unimodular, where $U_{i}=$ $D_{c} D+N_{c} N_{i}=U_{k}-\left[1-\Pi_{\ell=k+1}^{i}\left(1-\Delta_{\ell}\right)\right] N_{c} N_{k}$, i.e.,

$$
U_{i}=U_{k}-\Phi_{i} N_{c} N_{k} \text {. }
$$

By (32), $U_{i}$ is unimodular if and only if $U_{k}^{-1} U_{i}=$ $I-\Phi_{i} U_{k}^{-1} N_{c} N_{k}$ is unimodular, equivalently, $\hat{U}_{i}=I-$ $\Phi_{i} N_{k} U_{k}^{-1} N_{c}=I-\Phi_{i} T_{k} \quad$ is unimodular. Since $G_{k} H \in \mathcal{M}\left(\mathbf{R}_{s}\right) \quad$ implies $T_{k} \in \mathcal{M}\left(\mathbf{R}_{\mathbf{s}}\right)$, we have $s T_{k} \in \mathcal{M}(\mathbf{S})$. By (2) and (9), $s^{-1} \Delta_{i} \in \mathbf{S}$ implies $s^{-1} \Phi_{i}=$ $s^{-1} \Delta_{k+1}+\sum_{j=k+2}^{i} s^{-1} \Delta_{j} \Pi_{\ell=k+1}^{j-1}\left(1-\Delta_{\ell}\right) \in \mathbf{S}$. By (5), $\left\|s^{-1} \Phi_{i}\right\| \leq\left\|s^{-1} \Delta_{k+1}\right\|+\sum_{j=k+2}^{i}\left\|s^{-1} \Delta_{j} \Pi_{\ell=k+1}^{j-1}\left(1-\Delta_{\ell}\right)\right\|$ $\leq r_{k+1}+\sum_{j=k+2}^{i} r_{j} \Pi_{\ell=k+1}^{j-1} q_{\ell}=R_{k i} \leq R_{k \rho}$. If (13) holds, then $\quad\left\|\Phi_{i} T_{k}\right\|=\left\|\left(\Phi_{i} / s\right) s T_{k}\right\| \leq\left\|\Phi_{i} / s\right\|\left\|s T_{k}\right\| \leq R_{k 2} /$ $\left(\delta+R_{k \rho}\right)<1$ implies $\hat{U}_{i}=I-s^{-1} \Phi_{i} s T_{k}$ is unimodular, equivalently, $H$ also stabilizes $G_{i}$. To show (14)-(16), use (31) to write $T_{i}=N_{i} U_{i}^{-1} N_{c}=N_{i} U_{i}^{-1} U_{i-1} U_{i-1}^{-1} N_{c}=$ $N_{i} U_{i}^{-1}\left(U_{i}+\Delta_{i} N_{c} N_{i-1}\right) U_{i-1}^{-1} N_{c}=\left(1-\Delta_{i}\right) N_{i-1} U_{i-1}^{-1} N_{c}+$ $\Delta_{i} N_{i} U_{i}^{-1} \times \quad N_{c} N_{i-1} U_{i-1}^{-1} N_{c}$; use (32) to write $T_{i}=N_{i} U_{i}^{-1} U_{k} U_{k}^{-1} N_{c}=N_{i} U_{i}^{-1}\left(U_{i}+\Phi_{i} N_{c} N_{k}\right) U_{k}^{-1} N_{c}=$ $\Pi_{\ell=k+1}^{i}\left(1-\Delta_{\ell}\right) N_{k} U_{k}^{-1} N_{c}+\Phi_{i} N_{i} U_{i}^{-1} N_{c} N_{k} U_{k}^{-1} N_{c}$. Then

$$
T_{i}=\left(1-\Delta_{i}\right) T_{i-1}+\Delta_{i} T_{i} T_{i-1}=\left(1-\Phi_{i}\right) T_{k}+\Phi_{i} T_{i} T_{k} .
$$

Multiplying by $s, \quad\left\|s T_{i}\right\|=\| \Pi_{\ell=k+1}^{i}\left(1-\Delta_{\ell}\right) s T_{k}+$ $s^{-1} \Phi_{i} s T_{i} s T_{k}\left\|\leq\left(\Pi_{\ell=k+1}^{i} q_{\ell}+R_{k i}\left\|s T_{i}\right\|\right) \quad\right\| s T_{k} \|=(\delta+$ $\left.R_{k \rho}\right)^{-1}\left(\Pi_{\ell=k+1}^{i} q_{\ell}+R_{k i}\left\|s T_{i}\right\|\right) \quad$ implies $\quad\left(\delta+R_{k \rho}-R_{k i}\right)$ $\left\|s T_{i}\right\|_{\leq} \leq \prod_{\ell=k+1}^{i} q_{\ell} . \quad$ By $\quad(5), \quad R_{k \rho}-R_{k i}=\sum_{j=k+2}^{\rho} \times$ $r_{j} \Pi_{\ell=k+1}^{j-1} q_{\ell}-\sum_{j=k+2}^{i} r_{j} \Pi_{\ell=k+1}^{j-1} q_{\ell}=\sum_{j=i+1}^{\rho} r_{j} \Pi_{\ell=k+1}^{j-1} q_{\ell}=$ $\Pi_{\ell=k+1}^{i} q_{\ell}\left[r_{i+1}+\sum_{j=i+2}^{\rho} r_{j} \Pi_{\ell=i+1}^{j-1} q_{\ell}\right]=\prod_{\ell=k+1}^{i} q_{\ell} R_{i \rho}$. The bound on $\left\|s T_{i}\right\|$ follows from $\left(\delta+R_{k \rho}-R_{k i}\right)\left\|s T_{i}\right\|=$ $\left(\delta+\prod_{\ell=k+1}^{i} q_{\ell} R_{i \rho}\right)\left\|s T_{i}\right\| \leq \Pi_{\ell=k+1}^{i} q_{\ell} . \quad$ By $\quad(33), \quad\left\|T_{i}\right\| \leq$ $\left\|\left(1-\Delta_{i}\right) T_{i-1}\right\|+\left\|T_{i}\right\|\left\|s^{-1} \Delta_{i} s T_{i-1}\right\|$. But $\quad \|\left(1-\Delta_{i}\right)$ $T_{i-1} \| \leq \min \left\{\left\|1-\Delta_{i}\right\|\left\|T_{i-1}\right\|,\left\|T_{i-1}\right\|+\left\|s^{-1} \quad \Delta_{i} s T_{i-1}\right\|\right\}$, and $\left\|s^{-1} \Delta s T_{i-1}\right\| \leq r_{i} \alpha_{i}$ give the upper-bound on $\left\|T_{i}\right\|$ relative to $\left\|T_{i-1}\right\|$. The lower-bound follows from (33), with $T_{i-1}=T_{i}+\Delta_{i} T_{i-1}-\Delta_{i} T_{i} T_{i-1}$ implying $\left\|T_{i-1}\right\| \leq$ $\left\|T_{i}\right\|+\left(1+\left\|T_{i}\right\|\right)\left\|s^{-1} \Delta_{i}\right\|\left\|s T_{i-1}\right\|=\left(1+r_{i} \alpha_{i}\right)\left\|T_{i}\right\|+r_{i} \alpha_{i}$. Similarly from (33), $\quad\left\|T_{i}\right\| \leq\left\|\left(1-\Phi_{i}\right) T_{k}\right\|+$ $\left\|T_{i}\right\|\left\|s^{-1} \Phi_{i} s T_{k}\right\|$ implies the bounds on $\left\|T_{i}\right\|$ relative to $\left\|T_{k}\right\|$ by replacing $\Delta_{i}, r_{i}, \alpha_{i}, T_{i-1}$ with $\Phi_{i}, R_{k i}, \alpha_{k+1}, T_{k}$. For the bounds on $S_{i}$, from (33), $I-S_{i}=I-S_{i-1}-$ $\Delta_{i} T_{i-1}+\Delta_{i}\left(I-S_{i}\right) T_{i-1}=I-S_{k}-\Phi_{i} T_{k}+\Phi_{i}\left(I-S_{i}\right) T_{k}$ implies $S_{i}=S_{i-1}+\Delta_{i} S_{i} T_{i-1}=S_{k}+\Phi_{i} S_{i} T_{k}$. Finally, (15) follows from $\left\|S_{i-1}\right\|-r_{i} \alpha_{i}\left\|S_{i}\right\| \leq\left\|S_{i}\right\| \leq$ $\left\|S_{i-1}\right\|+r_{i} \alpha_{i}\left\|S_{i}\right\| \quad$ and $\quad\left\|S_{k}\right\|-R_{k i} \alpha_{k+1}\left\|S_{i}\right\| \leq\left\|S_{i}\right\| \leq$ $\left\|S_{k}\right\|+R_{k i} \alpha_{k+1}\left\|S_{i}\right\|$.

Proof of Lemma 2: Let $P=\tilde{D}^{-1} \tilde{N}$ be an LCF and let $\tilde{H}=\tilde{N}_{c} \tilde{D}_{c}^{-1}$ be an RCF. For $k \geq 0$, the controller $\tilde{H}$ stabilizes $P_{k}$ if and only if $V_{k}:=\tilde{D}_{k} \tilde{D}_{c}+\tilde{N} \tilde{N}_{c}$ is unimodular. By assumption, for some $k \geq 0, V_{k}$ is unimodular. We show that $\tilde{H}$ also stabilizes $P_{i}$ by induction: Suppose that $V_{i-1}$ is unimodular, which is already given for $i=k+1$. Then $\tilde{D}_{i}:=\left(1-\tilde{\Delta}_{i}\right) \tilde{D}_{i-1}$ implies

$$
\begin{aligned}
V_{i} & =\tilde{D}_{i} \tilde{D}_{c}+\tilde{N} \tilde{N}_{c}=\tilde{D}_{i} \tilde{D}_{c}+V_{i-1}-\tilde{D}_{i-1} \tilde{D}_{c} \\
& =V_{i-1}-\tilde{\Delta}_{i} \tilde{D}_{i-1} \tilde{D}_{c} .
\end{aligned}
$$

By (34), $V_{i}$ is unimodular if and only if $V_{i-1}^{-1} V_{i}=$ $I-V_{i-1}^{-1} \tilde{\Delta}_{i} \tilde{D}_{i-1} \tilde{D}_{c} \quad$ is unimodular, equivalently, $\tilde{V}_{i}:=1-\tilde{\Delta}_{i} \tilde{D}_{c} V_{i-1}^{-1} \tilde{D}_{i-1}=I-\tilde{\Delta}_{i} S_{i-1}$ is unimodular. Since $P_{k} \tilde{H}$ is type-1 or greater, we have $S_{i-1}(0)=0$ for $i \geq k+1$, and consequently, $\left(s^{-1} S_{i-1}\right) \in \mathcal{M}(\mathbf{S})$. By (6), $s \tilde{\Delta}_{i} \in \mathbf{S}$. By (3), if condition (25) holds, then $\left\|s \tilde{\Delta}_{i} s^{-1} S_{i-1}\right\| \leq\left\|s \tilde{\Delta}_{i}\right\|\left\|s^{-1} S_{i-1}\right\|=r_{i}\left\|s^{-1} S_{i-1}\right\|<1$. 
Therefore, $\tilde{V}_{i}=I-s \tilde{\Delta}_{i} s^{-1} S_{i-1}$ is unimodular, equivalently, $\tilde{H}$ stabilizes $P_{i}$.

Proof of Theorem 2: The proof uses entirely similar steps as the proof of Theorem 1 and follows from the transformation $s \rightarrow s^{-1}$ : For $k+1 \leq i \leq \rho, \tilde{H}$ stabilizes $P_{i} \quad$ if and only if $V_{i}=\tilde{D}_{i} \tilde{D}_{c}+\tilde{N} \tilde{N}_{c}=$ $V_{k}-\left[1-\Pi_{\ell=k+1}^{i}\left(1-\tilde{\Delta}_{\ell}\right)\right] \tilde{D}_{k} \tilde{D}_{c}=V_{k}-\tilde{\Phi}_{i} \tilde{D}_{k} \tilde{D}_{c}$ in $(34)$ is unimodular if and only if $V_{k}^{-1} V_{i}=I-\tilde{\Phi}_{i} V_{k}^{-1} \tilde{D}_{k} \tilde{D}_{c}$ is unimodular, equivalently, $V_{i}=I-$ $\tilde{\Phi}_{i} \tilde{D}_{c} V_{k}^{-1} \tilde{D}_{k}=I-\tilde{\Phi}_{i} S_{k}$ is unimodular. Since $P_{k} \tilde{H}$ is type-1 or greater, i.e., $S_{k}(0)=0$, we have $\left(s^{-1} S_{k}\right) \in \mathcal{M}(\mathbf{S})$. By (6) and (23), $s \tilde{\Delta}_{i} \in \mathbf{S}$ implies $s \tilde{\Phi}_{i}=s \tilde{\Delta}_{k+1}+\sum_{j=k+2}^{i} s \tilde{\Delta}_{j} \Pi_{\ell=k+1}^{j-1}\left(1-\tilde{\Delta}_{\ell}\right) \in \mathbf{S}$. By (5), $\left\|s \tilde{\Phi}_{i}\right\| \leq\left\|s \tilde{\Delta}_{k+1}\right\|+\sum_{j=k+2}^{i}\left\|s \tilde{\Delta}_{j} \Pi_{\ell=k+1}^{j-1} \quad\left(1-\tilde{\Delta}_{\ell}\right)\right\| \leq$ $r_{k+1}+\sum_{j=k+2}^{i} r_{j} \Pi_{\ell=k+1}^{j-1} q_{\ell}=R_{k i} \leq R_{k \rho}$. If (26) holds, then $\quad\left\|\tilde{\Phi}_{i} S_{k}\right\|=\left\|s \tilde{\Phi}_{i} s^{-1} S_{k}\right\| \leq \quad\left\|s \tilde{\Phi}_{i}\right\|\left\|s^{-1} S_{k}\right\| \leq$ $\left(R_{k i} /\left(\delta+R_{k \rho}\right)\right)<1$ implies $\hat{V}_{i}=I-\tilde{\Phi}_{i} S_{k}$ is unimodular, equivalently, $\tilde{H}$ also stabilizes $P_{i}$. To show (27)-(29), write $\quad S_{i}=\tilde{D}_{c} V_{i}^{-1} \tilde{D}_{i}=\tilde{D}_{c} V_{i-1}^{-1} V_{i-1} V_{i}^{-1} \tilde{D}_{i}=\tilde{D}_{c} V_{i-1}^{-1}$ $\left(V_{i}+\tilde{\Delta} \tilde{D}_{i} \tilde{D}_{c}\right) V_{i}^{-1} \tilde{D}_{i} \quad=\tilde{D}_{c} V_{i-1}^{-1} \tilde{D}_{i}+\tilde{\Delta} \tilde{D}_{c} V_{i-1}^{-1} \tilde{D}_{i-1} \tilde{D}_{c}$ $V_{i}^{-1} \tilde{D}_{i}, S_{i}=\tilde{D}_{c} V_{k}^{-1} V_{k} V_{i}^{-1} \tilde{D}_{i}=\tilde{D}_{c} V_{k}^{-1}\left(V_{i}+\tilde{\Phi} \tilde{D}_{k} \tilde{D}_{c}\right) V_{i}^{-1}$ $\tilde{D}_{i}=\tilde{D}_{c} V_{k}^{-1} \tilde{D}_{i}+\tilde{\Phi} \tilde{D}_{c} V_{k}^{-1} \tilde{D}_{k} \tilde{D}_{c} V_{i}^{-1} \tilde{D}_{i}$, i.e.,

$$
S_{i}=\left(1-\tilde{\Delta}_{i}\right) S_{i-1}+\tilde{\Delta} S_{i-1} S_{i}=(1-\tilde{\Phi}) S_{k}+\tilde{\Phi} S_{k} S_{i} .
$$

Multiplying by $\quad s^{-1}, \quad\left\|s^{-1} S_{i}\right\|=\|(1-\tilde{\Phi}) s^{-1} S_{k}+$ $s \tilde{\Phi} s^{-1} S_{k} s^{-1} S_{i}\left\|\leq\left(\Pi_{\ell=k+1}^{i} q_{\ell}+R_{k i}\left\|s^{-1} S_{i}\right\|\right) \quad\right\| s^{-1} S_{k} \|=$ $\left(\delta+R_{k \rho}\right)^{-1}\left(\Pi_{\ell=k+1}^{i} q_{\ell}+R_{k i}\left\|s^{-1} S_{i}\right\|\right)$ implies $\left(\delta+R_{k \rho}-\right.$ $\left.R_{k i}\right)\left\|s^{-1} S_{i}\right\| \leq \prod_{\ell=k+1}^{i} q_{\ell}$. The bound on $\left\|s^{-1} S_{i}\right\|$ follows from $\left(\delta+R_{k \rho}-R_{k i}\right)\left\|s^{-1} S_{i}\right\|=\left(\delta+\prod_{\ell=k+1}^{i} q_{\ell} R_{i \rho}\right)\left\|s^{-1} S_{i}\right\|$ $\leq \Pi_{\ell=k \pm 1}^{i} q_{\ell} . \quad$ By $\quad(35), \quad\left\|S_{i}\right\| \leq\left\|\left(1-\tilde{\Delta}_{i}\right) S_{i-1}\right\|+$ $\left\|S_{i}\right\|\left\|S \Delta_{i} s^{-1} S_{i-1}\right\|$. The upper-bound on $\left\|S_{i}\right\|$ relative to $\left\|S_{i-1}\right\|$ follows from $\left\|\left(1-\tilde{\Delta}_{i}\right) S_{i-1}\right\| \leq \min \left\{\left\|1-\tilde{\Delta}_{i}\right\|\right.$ $\left.\left\|S_{i-1}\right\|,\left\|S_{i-1}\right\|+\left\|s \tilde{\Delta}_{i} S^{-1} S_{i-1}\right\|\right\}$, and $\left\|s \tilde{\Delta}_{i} s^{-1} S_{i-1}\right\| \leq r_{i} \beta_{i}$. The lower-bound follows from $S_{i-1}=S_{i}+\tilde{\Delta}_{i} S_{i-1}-$ $\tilde{\Delta}_{i} S_{i} S_{i-1} \quad$ implying $\quad\left\|S_{i-1}\right\| \leq\left\|S_{i}\right\|+\quad\left(1+\left\|S_{i}\right\|\right)\left\|s \tilde{\Delta}_{i}\right\|$ $\left\|S^{-1} S_{i-1}\right\|=\left(1+r_{i} \beta_{i}\right) \quad\left\|S_{i}\right\|+r_{i} \beta_{i}$. Similarly by (35), $\left\|S_{i}\right\| \leq\left\|\left(1-\tilde{\Phi}_{i}\right) S_{k}\right\|+\left\|S_{i}\right\| \quad\left\|s \tilde{\Phi}_{i} S^{-1} S_{k}\right\|$ implies the bounds on $\left\|S_{i}\right\|$ relative to $\left\|S_{k}\right\|$ follow by replacing $\tilde{\Delta}_{i}$, $r_{i}, \quad \beta_{i}, \quad S_{i-1}$ with $\tilde{\Phi}_{i}, \quad R_{k i}, \quad \beta_{k+1}, \quad S_{k}$. By (35), $I-T_{i}=I-T_{i-1}-\tilde{\Delta}_{i} S_{i-1}+\tilde{\Delta}_{i} S_{i-1}\left(I-T_{i}\right)=I-T_{k}-$ $\tilde{\Phi}_{i} S_{k}+\tilde{\Phi}_{i} S_{k}\left(I-T_{i}\right) \quad$ implies $\quad T_{i}=T_{i-1}+\tilde{\Delta}_{i} S_{i-1} T_{i}=$ $T_{k}+\tilde{\Phi}_{i} S_{i} T_{k}$. Finally, (29) follows from $\left\|T_{i-1}\right\|-r_{i} \beta_{i}$ $\left\|T_{i}\right\| \leq\left\|T_{i}\right\| \leq\left\|T_{i-1}\right\|+r_{i} \beta_{i}\left\|T_{i}\right\|$ and $\left\|T_{k}\right\|-R_{k i} \beta_{k+1} \times$ $\left\|T_{i}\right\| \leq\left\|T_{i}\right\| \leq\left\|T_{k}\right\|+R_{k i} \beta_{k+1}\left\|T_{i}\right\|$.

\section{References}

V.M. Adamjan, D.Z. Arov and M.G. Krein, "Analytic properties of Schmidt pairs for Hankel operator and the generalized SchurTakagi problem", Math. USSR Sbornik, 15, pp. 31-73, 1971.
A.C. Autoulas, D.C. Sorensen and S. Gugercin, "A survey of model reduction methods for large-scale systems", Contemporary Mathematics, 280, pp. 193-219, 2001.

U.M. Al-Saggaf and G.F. Franklin, "Model reduction via balanced realizations: an extension and frequency weighting techniques", IEEE Trans. Automat. Cont., 33, pp. 687-692, 1988.

B.D.O. Anderson and Y. Liu, "Controller reduction: concepts and approaches", IEEE Trans. Automat. Cont., 34, pp. 802-812, 1989.

D.S. Bernstein and D.C. Hyland, "The optimal projection equations for fixed-order dynamic compensation", IEEE Trans. Automat. Cont., 29, pp. 1034-1037, 1985.

J.C. Doyle, K. Glover, P.P. Khargonekar and B.A. Francis, "Statespace solutions to standard $\mathrm{H}_{2}$ and $H_{\infty}$ control problems", IEEE Trans. Automat. Cont., 34, pp. 831-847, 1989.

J.C. Doyle and G. Stein, "Multivariable feedback design: concepts for a classical/modern design", IEEE Trans. Automat. Cont., 26, pp. 4-16, 1981.

D.F. Enns, "Model reduction with balanced realizations: an error bound and a frequency weighted generalization", Proc. 23rd Conf. Decision Contr., Las Vegas, NV, pp. 127-132, 1984.

B.A. Francis, $A$ Course in $H_{\infty}$ Control Theory, New York: SpringerVerlag, 1987.

B.A. Francis and G. Zames, "On $H^{\infty}$-optimal sensitivity theory for SISO feedback systems", IEEE Trans. Automat. Cont., 29, pp. 9-16, 1984.

K. Glover, "All optimal Hankel-norm approximations of linear multivariable systems and their $L_{\infty}$-error bounds", Int. J. Cont., 39, pp. 1115-1193, 1984.

A.N. Gündeş and M.G. Kabuli, "Simultaneously stabilizing controller design for a class of MIMO systems", Automatica, 37, pp. 1989-1996, 2001.

S.Y. Kung and D.W. Lin, "Optimal Hankel-norm model reductions: multivariable systems", IEEE Trans. Automat. Cont., 26, pp. 832-852, 1981.

B.C. Kuo, Automatic Control Systems, 7th edition, New Jersey: Prentice Hall, 1995.

Y. Liu, B.D.O. Anderson and U.L. Ly, "Coprime factorization controller reduction with Bezout identity induced frequency weighting", Automatica, 26, pp. 233-249, 1990.

U.L. Ly, "A design algorithm for robust low-order controller". PhD Dissertation, Dep. Aeronaut. Asronaut, Stanford University, Stanford, CA (1982).

B.C. Moore, "Principal component analysis in linear systems: controllability, observability, and model reduction", IEEE Trans. Automat. Cont., 26, pp. 17-32, 1981.

K. Ogata, Modern Control Engineering, 3rd ed., New Jersey: Prentice Hall, 1997.

A.B. Özgüler and A.N. Gündeş, "Approximations in compensator design: a duality", Electronics Letters, 38, pp. 489-490, 2002.

A.B. Özgüler and A.N. Gündeş, "Plant order reduction for controller design"Proc. American Control Conference ACC'03, Denver Co., pp. 89-94, 2003.

C.E. Rohrs, J.L. Melsa and D.G. Schultz, Linear Control Systems, New Jersey: McGraw Hill, 1993.

M.C. Smith and K.P. Sondergeld, "On the order of stable compensators", Automatica, 22, pp. 127-129, 1986.

A. Varga, "On frequency-weighted coprime factorization based controller reduction", in Proc. American Control Conference ACC'03, Denver Co., pp. 3892-3897, 2003.

P.M.R. Wortelboer, M. Steinbuch and O.H. Bosgra, "Iterative model and controller reduction using closed-loop balancing with application to a compact disk mechanism", Int. J. Robust and Nonlinear Control, 9, pp. 123-142, 1999.

A. Yousuff, D.A. Wagie and R. Skelton, "Linear system approximation via covariance equivalent realization", J. Math. Anal. Appl., 106, pp. 91-115, 1985.

K. Zhou and J. Chen, "Performance bounds for coprime factor controller reductions", Sys. Cont. Lett., 26, pp. 119-127, 1995.

K. Zhou, J.C. Doyle and K. Glover, Robust and Optimal Control, Upper Saddle River, NJ: Prentice-Hall, 1996. 